\title{
Coloboma of optic nerve with overlay of peripapillary retina
}

\author{
DAVID G. COGAN \\ From the National Eye Institute, National Institutes of Health, Department of Health, Education, and \\ Welfare, Bethesda, Maryland, USA
}

SUMMARY An eye that had the clinical appearance of a tumour of the nerve head was found on histological examination to have a congenitally large scleral canal with secondary buckling of the overlying retina.

In 1961 Pedler described the histopathology of one type of optic nerve coloboma which he believed to be unique and which suggested to him a primary fault in maturation of the posterior sclera. He stated it was as though 'the peripapillary retina, optic disc, lamina cribosa, and posterior choroid were all driven posteriorly through the resultant hiatus'. In the following year a similar traction of the peripapillary retina on to the nerve head was pictured by Hogan and Zimmerman (1962) under the heading of 'Dysplasia of the Optic Disc', and 2 additional cases (one in a foal) were reported from the Institute of Ophthalmology (London) (Rack and Wright, 1966; Garner and Griffiths, 1969). These cases were likened to the morning glory syndrome (Kindler, 1970) in having large scleral canals without lamina cribosa, but they differ in their formation of an epipapillary mass that may clinically simulate a neoplasm.

In view of the paucity of cases which have been available histopathologically and because of the possible confusion with an intraocular tumour, the following case is submitted as an additional illustration of the Pedler type of coloboma.

\section{Case report}

The patient was first seen at the age of 9 months by Dr Sumner Liebman, who noted a lobulated mass over the disc of the right eye. A curious conformation of the vessels suggested angiomatosis or glioma. The eye was thought to be blind, though the patient's age precluded confident evaluation. The other eye was normal. Neurological and radiographical exami-

Address for reprints: Dr David G. Cogan, National Eye Institute, National Institutes of Health, Department of Health, Education, and Welfare, Bethesda, Maryland 20014, USA nations revealed a suggestively large head and 'some abnormality of the cerebral ventricles' (not further documented), but no other abnormality. The other eye was entirely normal. Since the possibility of tumour could not be excluded the eye was enucleated. This was 20 years ago. In the intervening 20 years the patient has developed normally and is now a college student.

\section{PATHOLOGY}

The eye was fixed in formalin, opened in the horizontal plane, and embedded in celloidin. It was of normal size and configuration. On the eye being opened the only abnormality found was the lobulated tissue overlying and surrounding the nerve head (Fig. 1).

Microscopically the anterior segment was entirely normal, showing a mature configuration of the angle structures, normal iris and ciliary body, and a lens that was in place with no evidence of cataract formation.

In the posterior segment the only abnormality was in the nerve head and surrounding region. Here the retina was bunched up to three times the normal thickness (Fig. 2) and folded on itself as though it had been drawn towards the axis of the nerve head and buckled on itself (Fig. 3). Indeed, the macular region, identified by the abundance of ganglion cells, approximated to the edge of the disc.

A mass of loosely arranged vascular connective tissue occupied the central portion of the nerve head, that portion which normally represents the physiological cup (Fig. 4). This tissue is distinctly abnormal in human eyes-though it simulates what is normally present in rabbit eyes-and appears in the present case to have been responsible for the traction of the adjacent retina.

Except for this bunching up of the peripapillary 


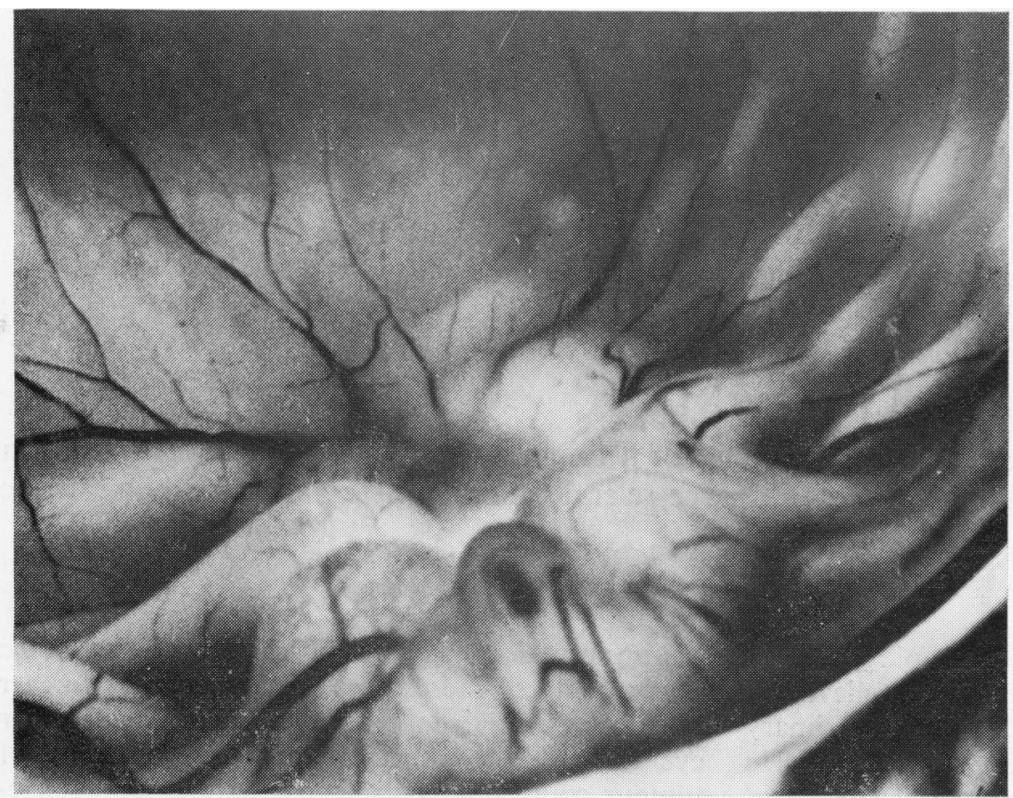

Fig. 1 Optic disc region of opened eye showing lobulated folds obscuring the disc margins

Fig. 2 Cross-section through nerve head showing buckling of retina and enlargement of the scleral canal. ( $H$ and $E \times 11)$

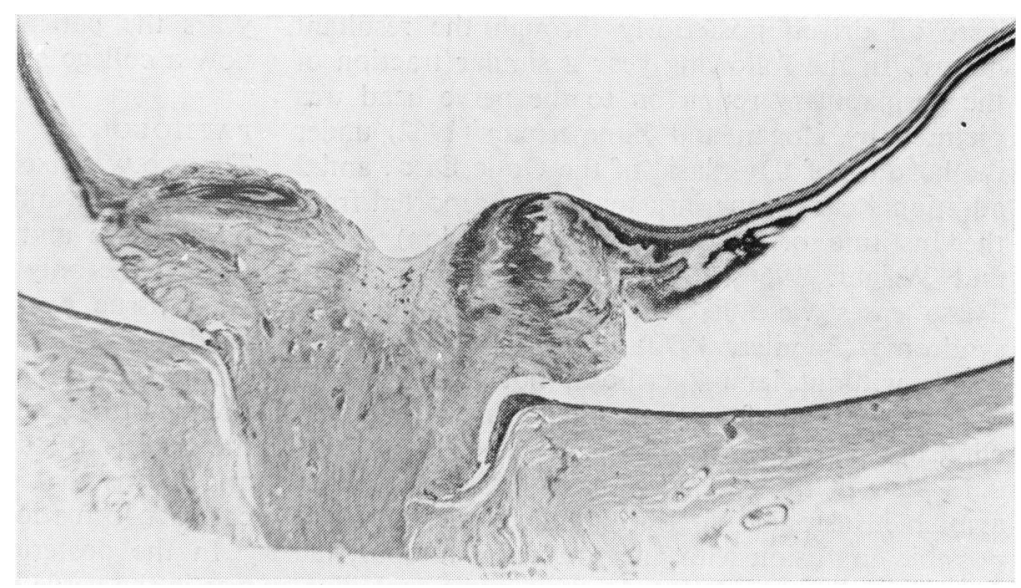

retina and its consequent local displacement the retina appeared normal and had obviously been in place during life. The choroid and sclera were also normal except for some funnelling of the latter posteriorly alongside the optic nerve.

Apart from buckling of the retina the other major abnormality was enlargement of the optic canal in the sclera and associated changes, including an absence or attenuation of the lamina cribosa, a border of gliotic tissue surrounding the nerve, and a further border of pigment epithelium adjacent to the sclera (Fig. 5). The scleral canal was approximately double the normal size. The optic nerve within this canal appeared normal, but the gliotic tissue on either side was distinctly abnormal. This tissue was randomly arranged and merged with the nerve without evidence of pia or arachnoid within the canal. The pigment epithelium appeared as a double layer in some areas with tubular lumina, and in other areas it appeared as hyperplastic pigment cells separated into sheets of metaplastic collagen. No other aberrant or ectopic tissue was present.

\section{Comment}

The patient presented with a mass overlying the optic disc suggestive of a tumour. It had a clinical 


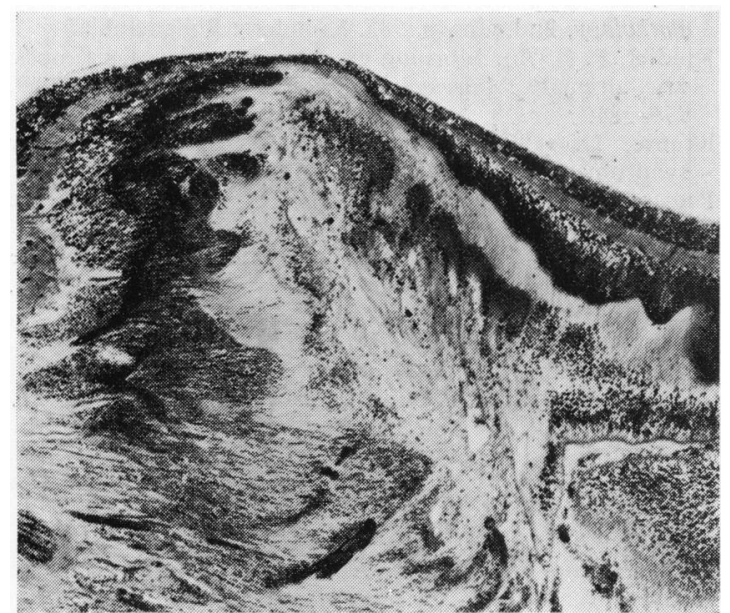

Fig. 3 Buckling of retina at edge of disc. Noteworthy is the mature, 3-layered differentiation of the retina with displacement of the macula (identified by the ganglion cells) towards the disc. ( $H$ and $E \times 34)$

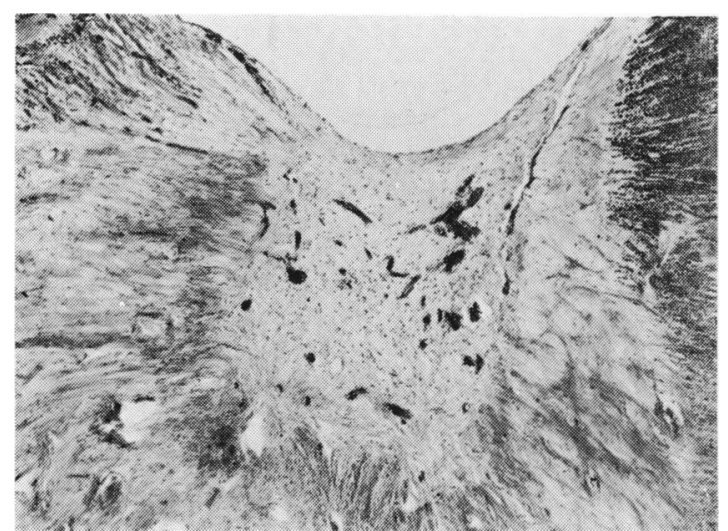

Fig. 4 Vascularised connective tissue in centre of nerve head. $(H$ and $E \times 32)$

appearance similar to that reported by Hamada and Ellsworth (1971) in patients with peripapillary detachment of the retina. On the other hand it did not have the ring of subretinal tissue about the excavated disc characteristic of the morning glory syndrome (Kindler, 1970; Krause, 1972).

Although Pedler's case (Pedler, 1961) is the pathological prototype of the anomaly represented by the present case, the most comprehensive histopathological study of relevant anomalies of the optic disc is that of Willis et al. (1972) with a report of 26 cases highlighting tissue in the region of the defect. Some of these cases were said to show mounds of gliosed retina filling the central area of the cup with layers of flattened and sometimes hyperplastic pigment epithelium behind the gliosed retina. Enucleation had been performed in 7 of these 27 cases because of suspected neoplasm. Details of the individual cases were not presented, but it seems altogether probable that some were similar to the present case.

The essential abnormalities in the present case consisted of: (1) a mass of loose, richly vascularised connective tissue in the region normally occupied by the physiological cup and possibly derived from the primitive hyaloid system; (2) secondary buckling of the peripapillary retina due presumably to the pull of the central connective tissue, so that the retina could not expand with the further growth of the rest of the eye; (3) large scleral canal with absence or defective formation of the lamina cribosa; and (4) presence of glial tissue and hyperplastic pigment epithelium between the optic nerve and sclera.

Although it is not apparent whether the enlargement of the scleral canal or the persistence of hyaloid tissue was the earlier and causative event, the buckling of the retina which gives the present case its

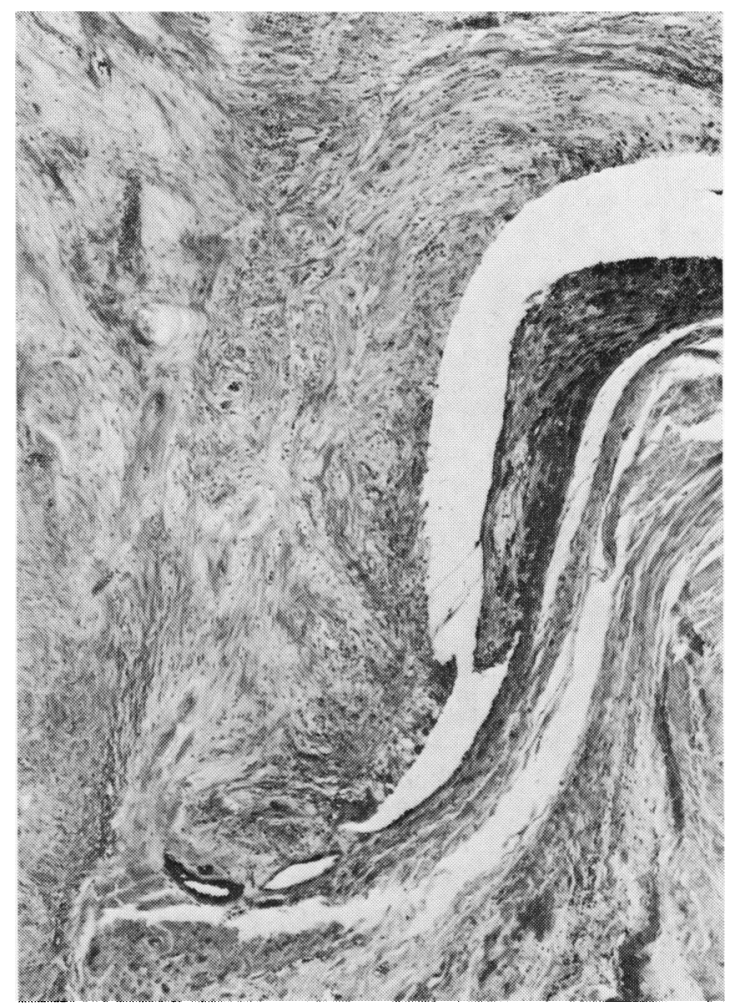

Fig. 5 Border tissue in scleral canal consisting (from left to right) of nerve trunk, glial tissue, artifactual cleft, hyperplastic pigment epithelium, and sclera. ( $H$ and $E$ $\times 45$ ) 
characteristic feature (and clinical importance) occurred relatively late, since the retina had developed its mature, 3-layered structure prior to the buckling.

\section{References}

Garner, A., and Griffiths, P. (1969). Bilateral congenital ocular defects in a foal. British Journal of Ophthalmology, 53, 513-517.

Hamada, S., and Ellsworth, R. M. (1971). Congenital retinal detachment and the optic disk anomaly. American Journal of Ophthalmology, 71, 460-464.

Hogan, M. J., and Zimmerman, L. E. (1962). Ophthalmic
Pathology, 2nd edn., p. 585. Saunders: Philadelphia.

Kindler, P. (1970). Morning glory syndrome: unusual optic disc anomaly. American Journal of Ophthalmology, 69, 376-384.

Krause, U. (1972). Three cases of the morning glory syndrome. Acta Ophthalmologica, 50, 188-198.

Pedler, C. (1961). Unusual coloboma of the optic nerve entrance. British Journal of Ophthalmology, 45, 803-807.

Rack, J. H., and Wright, G. F. (1966). Coloboma of the optic nerve entrance. British Journal of Ophthalmology, 50, 705-709.

Willis, R., Zimmerman, L. E., O'Grady, R., Smith, R. S. and Crawford, B. (1972). Heterotopic adipose tissue and smooth muscle in the optic disc. Archives of Ophthalmology, 88, 139-146. 